\title{
Periprosthetic Joint Infection in Hip Arthroplasty: Is There an Association Between Infection and Bearing Surface Type?
}

\author{
Rocco P. Pitto MD, PhD, Laurent Sedel MD
}

Published online: 1 June 2016

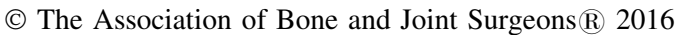

\begin{abstract}
Background Preliminary studies have raised the question of whether certain prosthetic biomaterials used in total hip arthroplasty (THA) bearings are associated with increased risk of periprosthetic joint infection (PJI). For example, some observational data suggest the risk of PJI is higher with metal-on-metal bearings. However, it is not known whether other bearings-including ceramic bearings or metal-on-polyethylene bearings - may be associated with a higher or lower risk of PJI.

Questions/purposes The objective of this study was to use a national arthroplasty registry to assess whether the choice of bearings-metal-on-polyethylene (MoP), ceramic-on-polyethylene $(\mathrm{CoP})$, ceramic-on-ceramic $(\mathrm{CoC})$, or metal-on-metal (MoM) -is associated with differences in the risk of revision for deep infection, either (1) within 6
\end{abstract}

One of the authors (RPP) received research support funding from CeramTec, Plochingen, Germany.

All ICMJE Conflict of Interest Forms for authors and Clinical Orthopaedics and Related Research $\mathbb{R}$ editors and board members are on file with the publication and can be viewed on request.

Each author certifies that his or her institution approved the human protocol for this investigation, that all investigations were conducted in conformity with ethical principles of research, and that informed consent for participation in the study was obtained.

This work was performed at Middlemore Hospital, University of Auckland, Auckland, New Zealand.

R. P. Pitto $(\square)$

Department of Orthopaedic Surgery, Middlemore Hospital, University of Auckland, South Auckland Clinical Campus, Auckland 93311, New Zealand

e-mail: r.pitto@auckland.ac.nz

L. Sedel

Lariboisière/Saint-Louis Hospital, University of Paris Denis Diderot, Paris, France months or (2) over the entire period of observation, which spanned 15 years.

Methods Data from primary THAs were extracted from the New Zealand Joint Registry over a 15-year period. A total of 97,889 hips were available for analysis. Inclusion criterion was degenerative joint disease; exclusion criteria were previous surgery, trauma, and any other diagnosis (12,566 hips). We also excluded a small group of ceramicon-metal THAs (429) with short followup. The median observation period of the selected group of hips $(84,894)$ was 9 years (range, $1-15$ years). The mean age of patients was 68 years ( $\mathrm{SD} \pm 11$ years), and $52 \%$ were women. There were 54,409 (64\%) MoP, 16,503 (19\%) CoP, 9051 (11\%) CoC, and 4931 (6\%) MoM hip arthroplasties. Four hundred one hips were revised for deep infection. A multivariate assessment was carried out including the following risks factors available for analysis: age, sex, operating room type, use of body exhaust suits, THA fixation mode, and surgeon volume. Because of late introduction of data collection in the Registry, we were unable to include body mass index (BMI, recording started 2010) and medical comorbidities according to the American Society of Anesthesiologists class (ASA, recording started 2005) in the multivariate analysis.

Results The rate of early PJI ( $<6$ months) did not differ by bearing surface. In contrast, we observed a difference over the total observation period. Within the first 6 months after the index surgery, CoC THAs were not associated with a lower risk of revision for PJI $(p=0.118)$ when compared with CoP (hazard ratio [HR], 1.31; 95\% confidence interval [CI], 0.50-3.41), MoP (HR, 2.10; CI, 0.914.82), and MoM (HR, 2.04; CI, 0.69-6.09). When the whole observation period was considered, $\mathrm{CoC}$ hips were associated with a lower risk of revision for deep infection when compared with CoP (HR, 1.30; CI, 0.78-2.18; p = 
Table 1. Host- and surgery-related variables included in the analysis (age, sex, operative room type, THA fixation mode, use of body exhausts, and surgeon volume*

\begin{tabular}{|c|c|c|c|c|c|c|c|c|c|c|c|c|c|}
\hline \multirow[t]{2}{*}{$\begin{array}{l}\text { Bearing } \\
\text { surface }\end{array}$} & \multicolumn{2}{|l|}{ Sex } & \multicolumn{2}{|c|}{ Age (years) } & \multicolumn{2}{|c|}{ Operating room } & \multicolumn{3}{|c|}{ THA fixation mode } & \multicolumn{2}{|c|}{$\begin{array}{l}\text { Operating room } \\
\text { suits }\end{array}$} & \multicolumn{2}{|c|}{$\begin{array}{l}\text { Surgeon } \\
\text { volume } \\
\text { (THAs/year) }\end{array}$} \\
\hline & Females & Males & $\leq 64$ & $>65$ & $\begin{array}{l}\text { No Laminar } \\
\text { Flow }\end{array}$ & $\begin{array}{l}\text { Laminar } \\
\text { flow }\end{array}$ & Cemented & Uncemented & Hybrid & $\begin{array}{l}\text { No } \\
\text { suits }\end{array}$ & $\begin{array}{l}\text { Space } \\
\text { suits }\end{array}$ & $\leq 49$ & $>50$ \\
\hline \multicolumn{14}{|l|}{$\mathrm{CoC}$} \\
\hline Number & 4278 & 4773 & 7028 & 2023 & 4245 & 4713 & 0 & 7106 & 1945 & 5155 & 3896 & 3485 & 5566 \\
\hline Percent & $47 \%$ & $53 \%$ & $78 \%$ & $22 \%$ & $48 \%$ & $52 \%$ & & $78 \%$ & $22 \%$ & $57 \%$ & $43 \%$ & $38 \%$ & $62 \%$ \\
\hline \multicolumn{14}{|l|}{$\mathrm{CoP}$} \\
\hline Number & 7885 & 8618 & 8805 & 7698 & 9643 & 6551 & 504 & 10,829 & 5169 & 11,936 & 4566 & 8287 & 8215 \\
\hline Percent & $48 \%$ & $52 \%$ & $53 \%$ & $47 \%$ & $59 \%$ & $41 \%$ & $3 \%$ & $66 \%$ & $31 \%$ & $72 \%$ & $28 \%$ & $50 \%$ & $50 \%$ \\
\hline \multicolumn{14}{|l|}{ MoM } \\
\hline Number & 1626 & 3305 & 4281 & 650 & 2553 & 2316 & 7 & 4468 & 441 & 3581 & 1335 & 1921 & 2995 \\
\hline Percent & $33 \%$ & $67 \%$ & $87 \%$ & $13 \%$ & $52 \%$ & $48 \%$ & $0.1 \%$ & $91 \%$ & $9 \%$ & $73 \%$ & $27 \%$ & $39 \%$ & $61 \%$ \\
\hline \multicolumn{14}{|l|}{$\mathrm{MoP}$} \\
\hline Number & 30,053 & 24,356 & 13,175 & 41,234 & 34,071 & 19,397 & 18,712 & 33,148 & 32,996 & 39,050 & 15,337 & 32,338 & 22,049 \\
\hline Percent & $55 \%$ & $45 \%$ & $24 \%$ & $76 \%$ & $61 \%$ & $39 \%$ & $22 \%$ & $39 \%$ & $39 \%$ & $72 \%$ & $28 \%$ & $59 \%$ & $41 \%$ \\
\hline
\end{tabular}

* All these measures differed among the four bearing surface groups $(\mathrm{p}<0.01)$. A Cox proportional hazards regression was used to produce adjusted hazard ratios and a logistic regression for the adjusted odds ratios. There were 9051 (10.7\%) ceramic-on-ceramic (CoC) THAs, 16,503 (19.4\%) ceramic-on-polyethylene (CoP) THAs, 4931 (5.8\%) metal-on-metal (MoM) THAs, and 54,409 (64.1\%) metal-on-PE (MoP) THAs. The Registry data were not available in some cases; therefore, the total count of some variable does not correspond to the number of all THAs included in the study $(84,894)$.

$0.01)$, MoP (HR, 1.75; CI, 1.07-2.86; p = 0.02), and MoM (HR, 2.12; CI, 1.23-3.65; p = 0.008).

Conclusions Our finding associating CoC THA bearings with a lower risk of infection after THA must be considered very preliminary, and we caution readers against attributing all of the observed difference to the bearing surface. It is possible that some or all of the observed difference associated with bearing type may have been driven by other factors such as ASA and BMI, which could not be included in our multivariate analysis, and so future registry studies on this topic must assess those variables carefully.

Level of Evidence Level III, therapeutic study.

\section{Introduction}

Periprosthetic joint infection (PJI) is a disastrous complication after THA [3, 10]. The 2015 New Zealand Joint Registry report identified PJI as the fifth most common reason for primary THA revision, accounting for $12 \%$ of THA revisions (452 of 3914 revisions); only dislocation, aseptic loosening of the acetabular component, aseptic loosening of the femoral component, and pain were more common [16]. Recently, the type of bearing surface and the potential association with infection have been the subject of investigation [6]. Limited observational data show a higher association of infection with metal-on-metal bearings than with other kinds [2].

Metal debris frequently causes periarticular soft tissue necrosis, and polyethylene debris causes a distinctive foreign body reaction [4]. Ceramic debris is believed to be more inert than other articular surfaces debris and causes very little reaction [8]. Ceramic bearings also have lower wear than any other bearing couple, so there is less periprosthetic debris and subsequent tissue reaction $[1,9,11,12]$.

The objective of this study was to use a national arthroplasty registry to assess whether the choice of bearings-metal-on-polyethylene (MoP), ceramic-onpolyethylene $(\mathrm{CoP})$, ceramic-on-ceramic $(\mathrm{CoC})$, or metalon-metal (MoM) - is associated with differences in the risk of revision for deep infection, either (1) within 6 months or (2) over the entire period of observation, which spanned 15 years.

\section{Materials and Methods}

We extracted data on all primary THAs between January 1, 1999, and December 31, 2014, from the New Zealand National Joint Registry. This registry has a hospital compliance rate of $98 \%$, and data are validated against the New Zealand Health Information Service. During this period of 
time, 97,889 primary THAs were carried out in New Zealand. The mean age of the patients undergoing primary THA was 68 years $(\mathrm{SD} \pm 11)$ and $52 \%$ were women. Only $4 \%$ of THAs were bilateral carried out under the same anesthetic. Surgeon grade was recorded from 2005 onward with the majority of THAs performed by a board-certified orthopaedic surgeon (87\%). The recording of the American Society of Anesthesiologists (ASA) physical status class was introduced at the beginning of 2005, and the recording of the body mass index (BMI) was introduced in 2010 . Because of the relatively small number of THAs with available ASA and BMI recording with followup longer than 6 months, we elected to exclude these two PJI risk parameters from the multivariate risk analysis.

\section{Participants/Study Subjects}

Inclusion criterion for this study was patients requiring primary THA as a result of noninflammatory arthritis (degenerative joint disease). Exclusion criteria were (1) secondary arthritis after avascular necrosis; (2) rheumatoid arthritis; (3) dysplasia; or (4) tumor. Moreover, we excluded patients with posttraumatic arthritis and/or previous surgery of the hip, both clinical conditions presenting an elevated risk of PJI. In total, 12,566 THAs carried out for diagnoses other than degenerative joint disease were excluded from the study. We also excluded a small singlesurgeon cohort of 429 THAs with ceramic-on-metal bearings and a short followup.

Complete data on 84,894 THAs were available for analysis. The median observation period was 9 years (range, 1-15 years). The bearing surfaces included 9051 (11\%) CoC, 16,503 (19\%) CoP, 4931 (6\%) MoM, and 54,409 (64\%) MoP combinations.

The choice of bearings may be influenced by patients and institutional factors; thus, we attempted to adjust the analysis and control for confounding variables like age and sex to isolate the effect of the bearing material on the outcome of interest. We also adjusted and controlled for the following potential risks factors for PJI available for analysis in the Registry database: operative room type (conventional versus laminar flow), fixation mode of THA (cemented, uncemented, hybrid), use of body exhaust systems, and surgeon volume. All these measures differed among the four bearing surface groups (Table 1). Therefore, we used Cox proportional hazards regression to produce adjusted hazard ratios and logistic regression for the adjusted odds ratios. In regard to the factor hospital volume, we note that most arthroplasty surgeons in New Zealand are practicing in both private and public settings. Therefore, we used surgeon volumes and type of operating room (a hospital factor) as factors for the multivariate analysis. The large majority (more than 90\%) of arthroplasties in New Zealand are carried out using 1 or $2 \mathrm{~g}$ cephalosporin preoperatively. Because of this rather homogeneous practice, we elected to exclude the factor preoperative antibiotic prophylaxis from multivariate analysis. Also, operative time was not used for analysis; a recent Registry study showed no association with the risk of revision for PJI in TKA [15].

\section{Statistical Analysis}

The cumulative proportion of patients revision-free for PJI of primary THAs at 6 months after the index surgery and after 6 months was estimated using the Kaplan-Meier method. The mortality rate of patients included in this study over the median of 9-year followup was $3 \%$. We therefore elected not to carry out a competing risks analysis. The primary endpoint was revision for PJI, rated acute if within 6 months postoperatively and late if revision occurred after the first 6 months after the index procedure. Odds ratios were calculated using the logistic regression and hazards ratios were calculated using the Cox proportional hazards regression analysis. The 95\% confidence intervals were calculated using a Poisson approximation. For all analyses, a two-tailed $\mathrm{p}$ value $<0.05$ was considered significant.

\section{Results}

After controlling for relevant confounding variables including age, sex, operative room type, use of body exhausts, THA fixation mode, and surgeon volume, we found no difference in risk of revision for deep infection within the first 6 months after the index THA in the four bearing surface cohorts $(\mathrm{CoC}$ hazard ratio [HR], 1, $\mathrm{p}=0.118$; $\mathrm{CoP} \mathrm{HR}, 1.31,95 \%$ confidence interval [CI], 0.50-3.41; MoP HR, 2.10, CI, 0.91-4.82; MoM HR, 2.04, CI, 0.69-6.09).

During the first 6 months after the index surgery $(80,618$ THAs with a minimum followup of 6 months), $0.07 \%$ (six of 8582 ) of CoC hips, $0.09 \%$ (14 of 15,273$)$ of CoP hips, $0.15 \%$ ( 76 of 51,862 ) of MoP hips, and $0.14 \%$ (seven of 4901) of MoM hips underwent revision for infection (Fig. 1). The rates of early infection appear lower than expected but do not include surgical procedures for PJI that did not require exchange of components and therefore were not recorded in the Registry.

In contrast, when the whole observation period was considered, $\mathrm{CoC}$ hips were associated with a lower risk of revision for infection (22 THAs, $p=0.013$ ) when compared with CoP (62 THAs; HR, 1.3; CI, 0.78-2.18), MoP (277 THAs; HR, 2.12; CI, 1.23-3.65), and MoM (40 
THAs; HR, 1.75; CI, 1.07-2.86) During the observation period of 15 years, a total of 401 of 84,894 THAs $(0.5 \%)$ were revised for PJI. One hundred three THAs of 401 (26\%) were revised for deep infection during the first 6 months postoperatively. The Kaplan-Meier survival analysis showed no revision for PJI after Year 10 after the index surgery in the $\mathrm{CoC}$ group of hips, whereas the other bearing surfaces groups showed a constant decrease of revision-free rates for PJI over time (Fig. 2).

\section{Discussion}

PJI is a serious and challenging complication in THA. Sir John Charnley in a presentation given to the Hip Society in

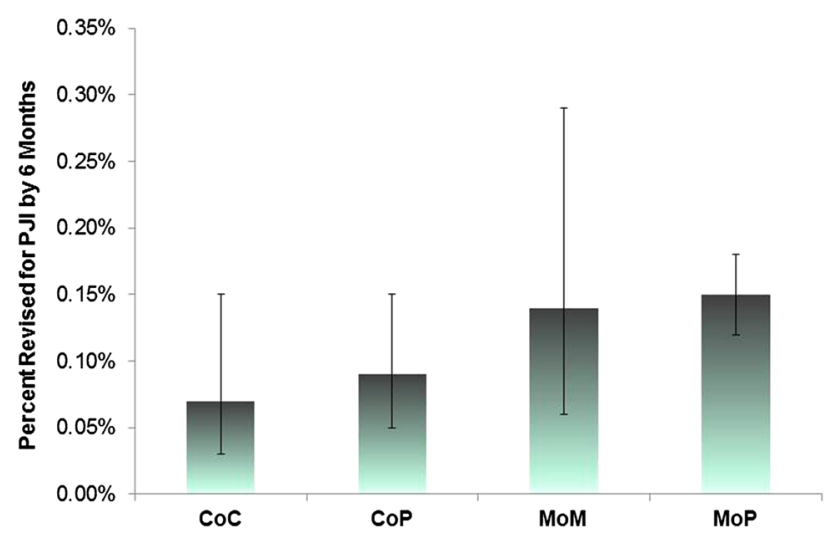

Fig. 1 This figure shows the percent of revision with CIs for PJI within 6 months after the index procedure by bearing surface.

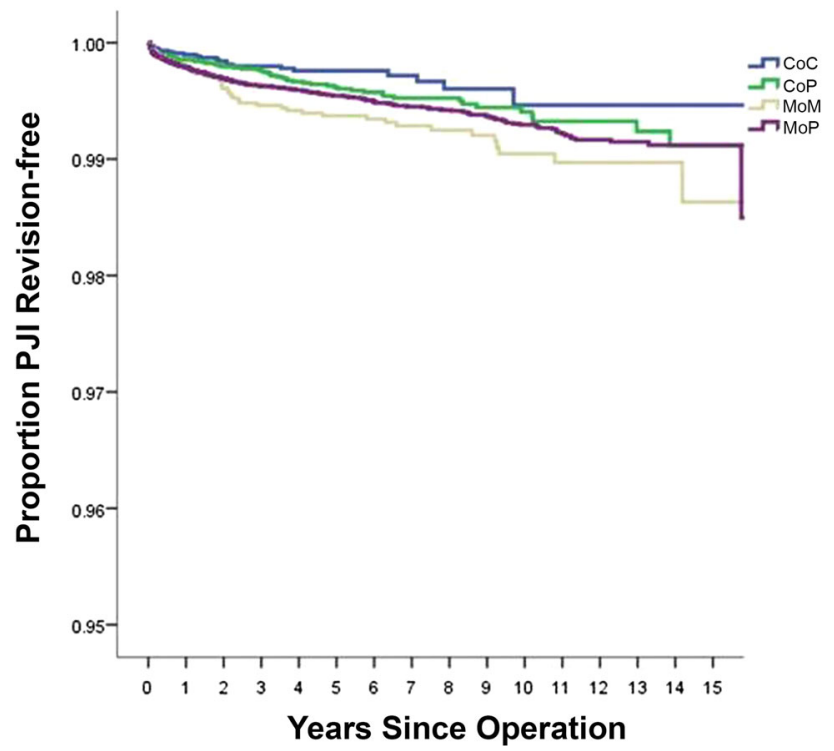

Fig. 2 The Kaplan-Meier survival analysis shows the proportion of revision-free THAs for PJI by bearing surface. The median observation period in this patient population $(84,894$ THAs) was 9 years (range, 1-15 years).
1982 stated: "Periprosthetic infection after total hip replacement is the saddest of all complications; it is sad because it seriously limits the success of any subsequent operation undertaken to revise a poor result following a first intervention .... I hope this demonstrates how very seriously I regard it as our duty to continue in the future to study to eliminate postoperative infection by any means, or combination of means, whatsoever" [3]. Studies have disagreed about whether bearing surface type is associated with a differential risk of PJI. Preliminary work suggests that it may be [2, 17], but to our knowledge, ceramic bearing surfaces have not been specifically studied in this way. We therefore asked whether the choice of bearings is associated with differences in the risk of revision for PJI, either within 6 months or over the entire period of observation of 15 years, using a national arthroplasty registry database. We found that the rate of early PJI did not differ by bearing surface. In contrast, when the whole observation period was considered, $\mathrm{CoC}$ hips were associated with a lower risk of revision for deep infection when compared with $\mathrm{CoP}, \mathrm{MoP}$, and MoM bearings.

This study had several relevant limitations. Host-related risk factors like poorly controlled diabetes, malnutrition, morbid obesity, chronic renal disease, active liver disease, excessive smoking, and/or alcohol consumption were not included in the multivariate analysis [10]. In particular, two factors that have been associated in other studies with an increased risk of infection-BMI and medical comorbidities (or a surrogate for them, like ASA class) - could not be included in our statistical model because the recording of the ASA physical status class was introduced at the beginning of 2005, and the recording of the BMI was introduced in 2010. As a result of the relatively small number of THAs with ASA and BMI recording with followup longer than 6 months, we elected to exclude these two PJI risk parameters from the multivariate risk analysis. This is potentially important, because in some studies, the effect size of the increased risk of infection associated with higher BMIs and ASA classes [10, 15] is comparable to the effect size we observed here in association with bearing surfaces. This suggests that some or all of the observed effect associated with bearing surface could have been the influence of those potentially confounding variables. However, we do not believe this is the case because in New Zealand, a small number of surgeons used and are regularly using $\mathrm{CoC}$ bearings for their patients ( 15 out of approximately 200 practicing surgeons during the whole observation period). Thus, most New Zealand patients requiring THA, including the lighter and healthier, are managed with $\mathrm{CoP}$ or $\mathrm{MoP}$ (and MoM in the past). In our opinion, this local feature excludes selection bias in the study.

Next, because this study was retrospective, we can only determine the presence of an association of revision for PJI 
and bearing surfaces; our study design does not allow us to attribute causation. In addition, we do not have information from the Registry regarding the microorganism causing the infection. Only large prospective multicenter studies will allow use of granular data for a comprehensive analysis.

The New Zealand Arthroplasty Registry data set is based on the clinical practice of a relatively small group of 213 orthopaedic surgeons with an average number of 39 THAs per surgeon carried out in 2014 [16]. During the 15year observation period, the use of crosslinked polyethylene continued its upward trend, making up $87 \%$ of the total polyethylene bearings in 2014. As a result of the marked discrepancy of median followup, we did not stratify the analysis according to polyethylene quality. Because of the relatively small sample size (401 revisions for PJI out of 84,894 hips), subgrouping the data set according to head diameter was considered not meaningful. We acknowledge that hips with large metal heads are more at risk of generating a milieu for PJI because of potential taper corrosion, whereas in contrast, ceramic heads can protect the patient from this complication [13, 14]. Analysis of bilateral THAs as independent observations is a potential statistical flaw of the study. However, only less than $4 \%$ of all THAs were performed under the same anesthetics and therefore probably using the same bearings and with the same exposure to associated PJI risks. On the other hand, the majority of bilateral THAs performed at different time points possibly had different bearing surfaces (different age of the patient and time of surgery, different surgeons and hospitals). Thus, we elected to analyze bilateral THAs as independent endpoints. The whole issue of surgical site infection is complex, and if ever there was good use for the word multifactorial, it would be to describe the possible causes of PJI. Adverse cellular reaction with inflammation and necrosis in the THA pseudocapsule can provide a favorable environment for bacterial proliferation, a potential reason as to why the incidence of PJI may be higher in THA with MoM bearings [6]. In contrast, the synovial-like pseudocapsule found in CoC THAs with fibrous tissue and limited inflammatory changes may potentially reduce the bioburden for late deep infection. However, this protective effect is merely hypothetical and derived from the literature [4, 5, 18].

The present Registry database assessment found a potential association between the bearing surface used during THA and deep infection. After adjusting for some (although admittedly not all) relevant confounding variables, CoC bearings seemed to be associated with a lower risk of revision for late PJI than other bearing couples. To our knowledge, this association has not been made before in large registry settings like ours. Although the association between several host-related and environmental risk factors is better understood today, the link between the use of different implant materials and PJI has not been clearly defined. Tokarski et al. [17] in a retrospective study involving 966 patients found that the use of tantalum acetabular components during revision THA was associated with a lower incidence of subsequent infection when used in patients with periprosthetic joint infection. Bozic et al. [2] in a large Medicare database of 148,827 patients undergoing THA found that MoM bearings were associated with higher risk of PJI than MoP and $\mathrm{CoC}$ bearings. A recent meta-analysis of five randomized clinical trials involving 897 patients with 974 hips and comparing reliability and durability of CoC (601) versus MoP (373) THAs showed that ceramic hips had decreased risks of revision for any reasons, but no difference was found between the two groups in regard to postoperative deep infection rate [7]. The total number of THAs included in the meta-analysis was relatively small considering the rate of revision for PJI is expected to be approximately $1 \%$. A power analysis related to PJI for this sample size was not reported in the article.

In conclusion, our finding associating CoC THA bearings with a lower risk of infection after THA must be considered very preliminary, and we caution readers against attributing all of the observed difference to the bearing surface. It is possible that some or all of the observed difference associated with bearing type may have been driven by other factors such as ASA and BMI, which could not be included in our multivariate analysis, and so future registry studies on this topic must assess those variables carefully. Multiinstitutional prospective studies that can collect granular data and assess the potential link between the bearing surface used and PJI in THA are currently underway.

Acknowledgments We thank Professor Chris Frampton, University of Otago, Christchurch, New Zealand, for the statistical analysis, and Tony Hobbs, New Zealand Arthroplasty Registry, Christchurch, New Zealand, for providing the database of this study. We also thank all New Zealand orthopaedic surgeons who are participating in the Hip Arthroplasty Registry.

\section{References}

1. Boutin P, Christel P, Dorlot J, Meunier A, de Roquancourt A, Blanquaert D, Herman S, Sedel L, Witvoet J. The use of dense alumina-alumina ceramic combination in total hip replacement. $J$ Biomed Mater Res. 1988;22:1203-1232.

2. Bozic KJ, Lau EC, Ong KL, Vail TP, Rubash HE, Berry DJ. Comparative effectiveness of metal-on-metal and metal-onpolyethylene bearings in Medicare total hip arthroplasty patients. J Arthroplasty. 2012;27:37-40.

3. Charnley J. The future of total hip replacement. In: Phillip NJ, ed. The Hip (Proceedings of the $10^{\text {th }}$ open scientific meeting of the Hip Society). St Louis, MO, USA: CV Mosby; 1982:198-210.

4. Esposito C, Maclean F, Campbell P, Walter WL, Walter WK, Bonar SF. Periprosthetic tissues from third generation alumina- 
on-alumina total hip arthroplasties. J Arthroplasty. 2013;28:860866.

5. Hernigou P, Homma Y, Pidet O, Guissou I, Hernigou J. Ceramicon-ceramic bearing decreases the cumulative long-term risk of dislocation. Clin Orthop Relat Res. 2013;471:3875-3882.

6. Hosman AH, van der Mei HC, Bulstra SK, Busscher HJ, Neut D. Effects of metal-on-metal wear on the host immune system and infection in hip arthroplasty. Acta Orthop. 2010;81:526-534.

7. Hu D, Tie K, Yang X, Tan Y, Alaidaros M, Chen L. Comparison of ceramic-on-ceramic to metal-on-polyethylene bearing surfaces in total hip arthroplasty: a meta-analysis of randomized controlled trials. J Orthop Surg Res. 2001;3:10-22.

8. Lerouge S, Huk O, Yahia LH, Witvoet J, Sedel L. Ceramicceramic and metal-polyethylene total hip replacements: comparison of pseudomembranes after loosening. J Bone Joint Surg Br. 1997;79:135-139.

9. Murphy SB, Ecker TM, Tannast M. Two- to 9-year clinical results of alumina ceramic-on-ceramic THA. Clin Orthop Relat Res. 2006;453:97-102.

10. Parvizi J, Gehrke T, Chen AF. Proceedings of the international consensus on periprosthetic joint infection. Bone Joint J. 2013;95:1450-1452.

11. Prudhommeaux F, Hamadouche M, Nevelos J, Doyle C, Meunier A, Sedel L. Wear of alumina-on-alumina total hip arthroplasties at a mean 11-year followup. Clin Orthop Relat Res. 2000;379:113-122.
12. Sedel L, Simeon J, Meunier A, Villette J, Launay S. Prostaglandin E2 level in tissue surrounding aseptic failed total hips. Arch Orthop Trauma Surg. 1992;111:255-258.

13. Sedel L, Walter WL, Pitto RP. Ceramic-on-ceramic THA: do the advantages outweigh the limitations? Clin Orthop Relat Res. 2014;472:2927-2931.

14. Tan SC, Lau AC, Del Balso C, Howard JL, Lanting BA, Teeter MG. Tribocorrosion: ceramic and oxidized zirconium vs cobaltchromium heads in total hip arthroplasty. J Arthroplasty. 2016 Feb 27 [Epub ahead of print].

15. Tayton ER, Frampton C, Hooper GJ, Young SW. The impact of patient and surgical factors on the rate of infection after primary knee arthroplasty: an analysis of 64,566 joints from the New Zealand Joint Registry Bone Joint J. 2016;98:334-340.

16. The New Zealand Joint Registry: Sixteen year report: January 1999 to December 2014. Available at: www.nzoa.org.nz/nz-jointregistry. Accessed May 2016.

17. Tokarski AT, Novack TA, Parvizi J. Is tantalum protective against infection in revision total hip arthroplasty? Bone Joint $J$. 2015;97:45-49.

18. Willert HG, Bertram H, Buchhorn GH. Osteolysis in alloarthroplasty of the hip: the role of ultra-high molecular weight polyethylene wear particles. Clin Orthop Relat Res. 1990;258:95-107. 